\title{
'Omics' of B. pseudomallei
}

\section{TJJ Inglis ${ }^{1,2}$}

Burkholderiapseudomallei, the cause of melioidosis, has been subjected to intense scrutiny across a range of 'Omics' systems from genome, to transcriptome, proteome and metabolomics analysis. The combination of two or more of these methods into a multi-layered approach is known as integrated systems biology. B. pseudomallei has been subject to extensive whole genome sequencing, its core genome extensively annotated and corresponding transcriptome explored. The rich pool of openly accessible $B$. pseudomallei genome data has clarified the molecular epidemiology, phylogenetic proximity of near-neighbour species and organisation of key operons.

Proteomic analysis with MALDI-TOF is now in use to assist laboratory identification of $B$. pseudomallei isolates from clinical samples, and has been used as an early warning in an outbreak investigation. The large genome of $B$. pseudomallei corresponds to an extensive repertoire of metabolic functions that enable bacterial adaptation to and survival in a wide range of environmental niches. The volatile organic compounds generated by active $B$. pseudomalle $i$ include sulphur compounds that explain the earthy, truffle-like odour of mature laboratory cultures. Single cell cytomic analysis of viable $B$. pseudomallei is at an early stage, but promises to set more mature genomic and other molecular analyses in a cellular context. The combination of these emerging analytical methods is expected to improve our understanding of disease pathogenesis, and open up new route to diagnosis, treatment and prevention.

\footnotetext{
${ }^{1}$ School of Pathology and Laboratory Medicine, University of Western Australia, Australia ${ }^{2}$ Department of Microbiology, PathWest Laboratory Medicine. Perth, Western Australia, Australia Address for correspondence: Prof TJJ Inglis, School of Pathology and Laboratory Medicine, University of Western Australia, Australia.+61407994631 Email: tim.inglis@uwa.edu.au ${ }^{10}$ https://orcid.org/0000-0002-8559-8245
} 